\title{
Evaluation of a High School Practical Experience on Noise Pollution With Smartphones
}

\author{
Anna R. Esteve ${ }^{1 \star}$, Sara Segura ${ }^{2}$, Jordi Solbes ${ }^{1}$ \\ ${ }^{1}$ Department of Experimental and Social Sciences Education. University of Valencia, SPAIN \\ 2 IES Ramón Llull, SPAIN
}

Received 8 April 2021 - Accepted 30 July 2021

\begin{abstract}
This work presents the evaluation of a practical experience on noise pollution based on the use of smartphones to check if it helps students to better understand the concepts related to this phenomenon. This evaluation has been done with a pre-post design in which 112 students, enrolled in the "Physics and Chemistry" course of the $1^{\text {st }}$ year of non-compulsory high school, have first filled in a diagnostics questionnaire with 5 free response questions about their knowledge on the topic and then a pre-post questionnaire with 4 free response questions. The results show that the proposed practical experience is effective since a statistically significant improvement is obtained both globally and in each of the questions of the questionnaire. The practical experience has also been highly valued by 38 students enrolled in the "Physics and Chemistry" specialisation of the Master in Secondary Education Teaching.
\end{abstract}

Keywords: smartphones, noise pollution, practical experience, classroom research, pre-post design

\section{INTRODUCTION}

In the education of responsible citizens, scientific literacy is fundamental, as well as for the socio-economic development of any country in the current globalized world (Hazelkorn et al., 2015). However, in recent years there has been a lack of interest for the science and technology subjects by the students, especially in physics and mathematics (Organisation for Economic Cooperation and Development, 2006; Potvin \& Hasni, 2014), which could lead to a lower research and innovation capacity in the future for many countries (Rocard et al., 2007).

During childhood, the students' attitude towards science is positive. However, their disinterest increases with age, and they end up dropping out the science subjects (physics, chemistry, or mathematics) in the upper years of high school (George, 2006; Murphy \& Beggs, 2006; Osborne et al., 1998; Simpson \& Oliver, 1990). Some of the causes of this disinterest are how science is usually taught, the negative image that students have of science (boring, difficult, useless and the cause of many environmental problems) or gender stereotypes (Osborne \& Dillon, 2008).

However, the student's motivation and interest to study science can benefit from certain teaching methodologies (for example, through inquiry-based learning or relating what is learned in school with their daily life, etc.) (Hasni \& Potvin, 2015). Therefore, since smartphones and/or tablets are usually available and students use them in their daily lives, nowadays there is the possibility of using these devices to carry out practical experiences in classrooms or laboratories. Practical experiences are essential to complement the conceptual contents, but not always the conditions in schools are ideal for carrying out traditional laboratory experiments. Therefore, physics and chemistry teachers can take advantage of the interest of their students in smartphones and tablets to propose practical experiences that use the sensors that these devices have (accelerometer, gyroscope, light sensor, etc.) as measurement tools both in the laboratory or classroom and in activities outside the classroom to increase their motivation (Kuhn \& Vogt, 2013a). Thus, students can see that what they learn in their Physics classes can be applied in their daily life (González et al., 2015), which

(c) 2021 by the authors; licensee Modestum. This article is an open access article distributed under the terms and conditions of the Creative Commons Attribution License (http://creativecommons.org/licenses/by/4.0/).

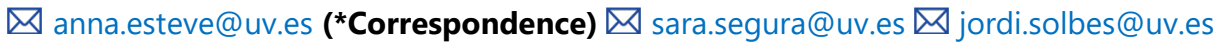




\section{Contribution to the literature}

- A practical experience on noise pollution based on the use of smartphones as measurement tools was designed to help students to better understand the concepts related to this phenomenon.

- The evaluation of the proposed practical experience with a pre-post questionnaire filled in by 112 students enrolled in the $1^{\text {st }}$ year of non-compulsory high school showed a statistically significant improvement in their learning about noise and noise pollution.

- The proposed practical experience was also highly valued by 38 students enrolled in the "Physics and Chemistry" specialisation of the Master in Secondary Education Teaching.

has a positive influence on their motivation (Kuhn \& Müller, 2015).

Although currently there are many proposals for practical experiences in which smartphones and/or tablets are used as measurement tools on mechanics (Hochberg et al., 2014; Vieyra \& Vieyra, 2014; Vogt \& Kuhn, 2012a, 2012b, 2014), acoustics (González \& González, 2016; Kuhn \& Vogt, 2013b; Kuhn et al., 2014; Parolin \& Pezzi, 2015, 2013) or optics (Sitar, 2012; Monteiro et al., 2017; Sans et al., 2017), there are few works that have included them in teaching proposals (Buongiorno et al., 2019) or tested the effectiveness of these in the students' learning of Physics (Esteve et al., 2019).

In the particular case of acoustics, despite its low presence in the physics curricula of high school, and sound often being treated as a particular case of waves, these contents are of great importance since they have applications in the life quality of people, their musical culture or the physiological knowledge of the human body. Thus, it would be necessary to include teaching proposals of physics that take advantage of the daily-life experiences of students to demonstrate the usefulness of these contents and overcome some of the difficulties students show with understanding this topic (Linder, 1992; Merino, 1998; Perales Palacios, 1997).

Therefore, the research goals of this work are the following:

1) Carry out a diagnostic of the knowledge about sound of students of the $1^{\text {st }}$ year of noncompulsory high school.

2) Design a practical experience on noise pollution based on the use of smartphones.

3) Check whether the proposed practical experience helps students of the $1^{\text {st }}$ year of non-compulsory high school to better understand the concepts related to this phenomenon, as well as check how it is valued by students enrolled in the "physics and chemistry" specialisation of the master in secondary education teaching.

In order to do this, a short diagnostics questionnaire was used to check the students' knowledge about sound. In addition, a sequence of activities has been carried out together with the practical experience based on the use of smartphones and a pre-post design has been carried out to evaluate whether it helps to improve the students' learning about this subject. Finally, this sequence of activities has been evaluated by graduates (and even doctors) in physics, chemistry, etc. who are enrolled in the "Physics and Chemistry" specialisation of the Master in secondary education teaching.

\section{METHODOLOGY}

\section{Context}

On the one hand, 112 students enrolled in the "physics and chemistry" course of the 1st year of noncompulsory high school (17-18 years old) filled in the diagnostics questionnaire on their previous knowledge about sound, did the practical experience and filled in the pre-post questionnaire in order to check whether it helps to improve their learning about noise and noise pollution.

On the other hand, 38 students enrolled in the "physics and chemistry" specialisation of the master in secondary education teaching filled in the questionnaire to evaluate this teaching proposal.

\section{Diagnostics of the High-School Students' Previous Knowledge}

In order to check the students' previous knowledge about sound, a diagnostic questionnaire was used. Table 1 shows its questions, some of which correspond to some of the categories used by Perales Palacios (1997) for the detection of the students' previous ideas on this topic: nature and description of sound, propagation of sound, and acoustic technology and applications. This questionnaire has five free-response questions: one about the nature of sound (D1), one about the propagation of sound (D2), one about the sound qualities that make it possible to distinguish sounds (D3), one about technological applications of sound (D4) and one about the perception of sound through the human ear (D5).

This questionnaire was answered before carrying out the practical experience and the students' answers were classified into the categories of complete, partially complete and incomplete according to the analysis criteria that are also shown in Table 1. For the statistical analysis of the questionnaire, the complete answers 
Table 1. Questions and analysis criteria of the diagnostic questionnaire

D1. Could you explain with your own words what sound is?

Complete Defining sound as a longitudinal wave or a pressure wave.

Partial Associating sound with a wave, without knowing which type it is, or stating that sound is a disturbance.

Incomplete Defining sound subjectively just as a sensation in the ear or not answering the question.

D2. In the Episode IV of Star Wars, Luke Skywalker blows up the enemy ship known as The Death Star, do you think

Luke heard the explosion of the Death Star just the same as he would have heard it on Earth?

Complete Stating that nothing can be heard in space since there is no medium that allows waves to travel.

Partial Stating that nothing can be heard in space, but not explaining the reason why.

Incomplete Stating that sound is heard in space just the same as on Earth or not answering the question.

D3. Could you distinguish the same note played on a piano and on a guitar? Or between the same note played on a trumpet and a violin? Why?

Complete Identifying timbre as what makes both instruments to have a different sound.

Partial Stating that both instruments can be distinguished by some property of sound, but not identifying timbre as the responsible.

Incomplete Stating that both instruments cannot be distinguished or that they can be distinguish by the sound intensity or frequency or not answering the question.

D4. Doctors often use a stethoscope to listen to the internal sounds of their patients such as their breathing or heartbeat. Could you explain how a stethoscope works?

Complete Stating that the stethoscope uses the transmission of sound through a medium to allow the internal sounds of the body to be heard.

Partial Relating the working of the stethoscope and the fact that sound is a longitudinal wave, but not explaining how the stethoscope works.

Incomplete Stating that the stethoscope amplifies the sound intensity or concentrates the sound waves or not answering the question.

D5. One of the most used tools to train dogs is an ultrasonic whistle, could you explain why this instrument does not disturb people? Or it does?

\begin{tabular}{ll}
\hline Complete & $\begin{array}{l}\text { Stating that ultrasound is outside the range of frequencies that can be heard by the human ear }(20 \mathrm{~Hz} \text { to } \\
20 \mathrm{kHz}) .\end{array}$ \\
Partial & $\begin{array}{l}\text { Relating it with the perception of sound through the ear, but not to the range of frequencies that can be } \\
\text { heard by the human ear. }\end{array}$ \\
Incomplete & Stating that ultrasound can be irritating for the human ear or not answering the question.
\end{tabular}

scored as 2, partially complete answers scored as 1 and incomplete answers scored as 0 .

\section{Practical Experience on Noise Pollution}

A practical experience on noise pollution has been designed following the one proposed by Science on Stage Germany (Andrade et al., 2014) and is based on the use of the smartphone's microphone and a specific app that allows access to the data recorded by this sensor. In this case, the Sound Meter app developed by Abc Apps has been used. This app, available for free for the Android operating system, measures the sound intensity level (SIL in $\mathrm{dB}$ ) that exists in a specific place at a given time.

The teaching proposal consists of 4 activities developed during 2 sessions of 1 hour each as well as outside the classroom. During the first session, before starting the practical experience, the basic concepts related to sound and noise pollution (definition and effects) are reviewed. The activities are described below:

\section{Activity 1. Calibration of the smartphone's sound meter}

After explaining how to use the sound meter app, students must test whether the average values recorded over $30 \mathrm{~s}$ with their smartphones coincide with those obtained by other classmates or not. This experience is used to introduce the concept of calibration and its importance when it comes to comparing measurements made with different devices. The calibration of all the smartphones' sound meter must be performed in a quiet spot in the school and using the YouTube video https://youtu.be/WJ9Go1PnAVA of pink noise (a "flat" or "even" sound to the human ear since all octave bands have the same sound level) as reference. Since the app should register a value of about $86 \mathrm{~dB}$ when keeping a distance of 1 meter between the smartphone and the speakers (power of 2-3 W) to the maximum volume level, students should keep their devices at this distance from the speakers and adjust the SIL measured by their smartphones with the "Calibrate" function of the app.

\section{Activity 2. Sound attenuation with distance}

Once students have become familiar with the use of the app and have calibrated their device, they check experimentally the sound attenuation with the square of the distance. To do this, students are asked first to estimate the SIL that is expected to be measured at certain distances from the speakers for the pink noise YouTube video. Next, students are asked to stand at 
Table 2. Questions and analysis criteria of the questionnaire for the evaluation of the students' learning

Q1. We use the term noise to refer to an unpleasant sound, do you think that living surrounded by noise can affect you? Why?

Complete Affirmative answer relating noise to health effects and psychological disorders.

Partial Affirmative answer but relating noise only to health effects or psychological disorders.

Incomplete Negative answer.

Q2. Which sources of noise pollution do you know?

Complete More than 2 sources of noise pollution.

Partial 1 or 2 sources of noise pollution.

Incomplete 1 source of noise pollution or none.

Q3. Which effects can noise pollution have?

Complete Examples related to auditory and non-auditory effects of noise.

Partial Examples related only to auditory or non-auditory effects of noise.

Incomplete Stating that noise pollution has no effect on humans.

Q4. What are decibels? Explain how they are related to sound.

Complete Defining decibels as a unit of measurement of sound intensity levels on a logarithmic scale and citing the human auditory range $(0-120 \mathrm{~dB})$.

Partial Defining decibels as a unit of measurement of sound intensity levels on a logarithmic scale or citing the human auditory range $(0-120 \mathrm{~dB})$.

Incomplete Not relating decibels to sound intensity levels.

those same distances from the speakers and record the mean value of the SIL measured over $30 \mathrm{~s}$ with their smartphones. Finally, students are asked to draw conclusions about the estimated and measured values, as well as on the sound attenuation with the distance to its emitting source.

\section{Activity 3. Sound map}

Students are asked to measure with their smartphones and record the mean value of the SIL over $30 \mathrm{~s}$ in different points within the school (classrooms, corridors, schoolyard, etc.) and its surrounding area (streets, parks, etc.) over the next few days at different times. These values will allow students to create a sound map of the school and its surrounding area.

\section{Activity 4. Group discussion}

Finally, to conclude the practical experience, a group discussion is held to draw conclusions about the results obtained in the sound map and the issue of noise pollution. To do this, students are asked the following questions: Which are the noisiest points and times within the school? Which are the noisiest points and times within the school's surrounding area? Could the school be considered a source of noise pollution? What measures could be taken to reduce noise levels within and around the school?

\section{Evaluation of the High-School Students' Learning Through the Practical Experience}

In order to check whether the proposed practical experience helps to improve the students' learning about noise and noise pollution, a pre-post design was used. Table 2 shows the four free-response questions of this questionnaire: one about the concept of noise pollution (Q1), one about its sources (Q2), one about its effects (Q3) and one about the decibel unit of measurement (Q4). The students' answers were classified into the categories of complete, partially complete and incomplete according to the analysis criteria that are also shown in Table 2.

This questionnaire was answered before carrying out the practical experience (pre-test) and after 15 days had elapsed (post-test). Thus, the results obtained in the posttest will be compared with those of the pre-test, both globally and question by question, to check if there are significant differences between the two. For the statistical analysis of the questionnaire, the complete answers scored as 2, partially complete answers scored as 1 and incomplete answers scored as 0 .

\section{Evaluation of the Practical Experience by Students of the Master in Secondary Education Teaching}

In order to have students of the "physics and chemistry" specialisation of the master in secondary education teaching to evaluate the proposed practical experience, the questionnaire shown in Table 3 was used. This questionnaire contains three scale questions (from 0 , strongly disagree, to 10 , strongly agree) and three free-response questions: one scale question about methodological aspects of the proposal (P1), one scale question about the contents of the proposal (P2), one free-response question about positive and negative aspects of the proposal (P3), two free-response questions about possible changes to the proposal (P4 and P5) and one scale question about the overall proposal (P6).

This questionnaire was answered after the Master's students carried out the practical experience during their training, in a single 2-hour session in which the basic concepts related to sound and noise pollution were reviewed, the smartphone's sound meter was calibrated, the sound attenuation with the square of the distance was checked, measurements of sound intensity levels 
Table 3. Questionnaire for the evaluation of the practical experience by students of the master in secondary education teaching

P1. Rate from 0 (strongly disagree) to 10 (strongly agree) the following methodological aspects of the practical experience on noise pollution with smartphones:

1) It helps to detect and correct errors.

2) It helps to acquire scientific knowledge.

3) It helps students to become familiar with scientific methodology.

4) It shows Science-Technology-Society (STS) relationships.

5) It encourages the students' participation.

6) It increases the students' interest in science.

P2. Rate from 0 (strongly disagree) to 10 (strongly agree) the following aspects about the contents of the practical experience on noise pollution with smartphones:

1) It takes into account the students' previous ideas about waves and sound.

2) It adequately presents the concept of noise pollution.

3) It links theory and practice.

4) It adequately explains the relationship between sound intensity levels measured at different distances.

5) It adequately presents the concept of decibel.

P3. Indicate other positive or negative aspects that you have found in this practical experience on noise pollution with smartphones.

P4. Which activities would you eliminate or change?

P5. What kind of activities would you use to replace the previous ones?

P6. Rate from 0 (strongly disagree) to 10 (strongly agree) your agreement with the following statements:

1) This topic can be eliminated from the Secondary Education curriculum.

2) The theory of this topic is very complicated.

3) This topic is not related with the students' reality.

4) This topic is not linked with the other Physics topics studied in the Secondary Education.

were made with smartphones in different points within the building and its surrounding area, and a group discussion was held to draw conclusions.

\section{RESULTS AND DISCUSSION}

\section{Diagnostics of the High-School Students' Previous Knowledge}

The diagnostic questionnaire has five questions with complete (2), partially complete (1) and incomplete (0) answers, so its maximum score is 10 and its minimum score is 0 . In this case, the global mean obtained by the students enrolled in the "Physics and Chemistry" course of the $1^{\text {st }}$ year of non-compulsory high school is 3.1 (with a standard deviation, SD, of 1.7), which indicates that they have a poor previous knowledge about sound.

Figure 1 shows the percentages of complete, partially complete and incomplete answers given by the students in the diagnostic questionnaire. The best results are obtained for the questions about the propagation of sound (D2) and the perception of sound through the human ear (D5). In question D2, 20.5\% of students give a complete answer stating that sound needs a medium to travel through (e.g., "Luke wouldn't hear the explosion because sound cannot travel in space since there's no air in it") and $78.6 \%$ state that nothing can be heard in space, although they do not know to explain why (partially complete answers such as "He didn't hear it because sound doesn't travel in space"). In question D5, 20.5\% of students know that ultrasound is outside the range of frequencies that can be heard by the human ear (e.g., "That whistle produces sounds with frequencies so high that people cannot hear them") and $43.8 \%$ of students know that ultrasound cannot be detected by humans, although they cannot relate it to the range of frequencies that can be heard by the human ear (e.g., "The whistle doesn't disturb people because humans cannot hear its sounds"). In the question about the nature of sound (D1), $78.6 \%$ of students associate sound with a wave, although without specifying which type it is (e.g., "Sound is a vibration that propagates as a wave"), with only $2.7 \%$ specifying that sound is a longitudinal or pressure wave (e.g., "Sound is the transmission of waves by the vibration of air molecules that can be perceived through the ear"). In the question about the sound qualities that make it possible to distinguish sounds (D3), $41.1 \%$ of students state that sounds produced by different musical instruments can be distinguish by some property of sound, but they are unable to specify which one (e.g., "The sound of each instrument is different even if it is the same note"), and only $6.3 \%$ of students know that timbre is the sound quality that makes it possible to distinguish the same note when played by different musical instruments (e.g., "It sounds differently because, although the note is the same, the timbre of both instruments is different"). The worst results are obtained for the question about the technological applications of sound (D4) since $76.8 \%$ of students are not able to relate the propagation of sound through a medium with the operation principle of a stethoscope (e.g., "It receives the heart's vibrations and transforms them into sound"). 




Figure 1. Percentage of answers given by the students in the diagnostic questionnaire

\section{Evaluation of the High-School Students' Learning Through the Practical Experience}

The questionnaire used in the pre-post design has 4 questions with complete (2), partially complete (1) and incomplete ( 0$)$ answers, so its maximum score is 8 and its minimum score is 0 . In this case, the global mean obtained by the students enrolled in the "Physics and Chemistry" course of the $1^{\text {st }}$ year of non-compulsory high school in the pre-test is $2.9(\mathrm{SD}=1.4)$ and $4.5(\mathrm{SD}=$ 1.4) in the post-test. Therefore, a slight increase is observed in the global score obtained by the students in the questionnaire after carrying out the practical experience on noise pollution based on the use of smartphones. However, statistical tests should be carried out to check if these differences between the global means of the pre-test and the post-test are statistically significant.

Since the samples are paired (pre-post design) and do not follow a normal distribution (according to the Kolmogorov-Smirnov test, with $\mathrm{p}<0.05)$, the nonparametric Wilcoxon signed-rank test was used, with the following result: $Z=-7.508, p<0.05$. Therefore, there are statistically significant differences between the global means of the pre-test and the post-test, so there has been a significant improvement when carrying out the practical experience on noise pollution based on the use of smartphones. Furthermore, a value of 0.5 is obtained for the size effect, which indicates a large effect.

Figure 2 shows the percentages of complete, partially complete and incomplete answers given by the students in the pre-post questionnaire. In the question about the concept of noise pollution (Q1), the percentage of incomplete and partially complete answers (e.g., "Yes, because it can cause stress") decrease in the post-test (from $14.3 \%$ to $3.6 \%$ and from $68.8 \%$ to $57.1 \%$, respectively), thus increasing the percentage of complete answers (such as "Yes, because it can increase the stress level, as well as producing hearing loss", from $17 \%$ to $39.3 \%$ ). In the question about sources of noise pollution (Q2), the percentage of incomplete answers (e.g., "cars" or "traffic") decreases considerably in the post-test (from $37.5 \%$ to $9.8 \%$ ) while the percentage of partially complete answers (e.g., "traffic, roadworks") decreases slightly (from $19.6 \%$ to $18.8 \%$ ), thus increasing the percentage of complete answers (such as "cars, loud music, airports, busy streets...", from $42.9 \%$ to $71.4 \%$ ). In the question about the effects of noise pollution (Q3), the percentage of incomplete answers (e.g., "I don't know") decreases considerably in the post-test (from $34.8 \%$ to $8.9 \%$ ), thus increasing the percentage of complete answers (such as "It can cause headaches, insomnia, dizziness, and hearing loss", from $5.4 \%$ to $26.8 \%$ ) and the percentage of partially complete answers (such as "It may cause hearing loss" or "It causes stress", from 59.8\% to $64.3 \%$ ). In the question about the decibel unit of measurement (Q4), the percentage of incomplete answers (e.g., "Decibels measure the number of vibrations per second that a wave has" or " $d B$ are the units of the speed of sound") decreases in the posttest (from 92\% to 67\%), increasing considerably the percentage of partially complete answers (such as "Decibels are the unit of measurement of sound intensity on a logarithmic scale", from $8 \%$ to $32.1 \%$ ) although the percentage of complete answers (e.g., "Decibels measure the sound intensity, which humans hear from 0 to $120 \mathrm{~dB}$ on 


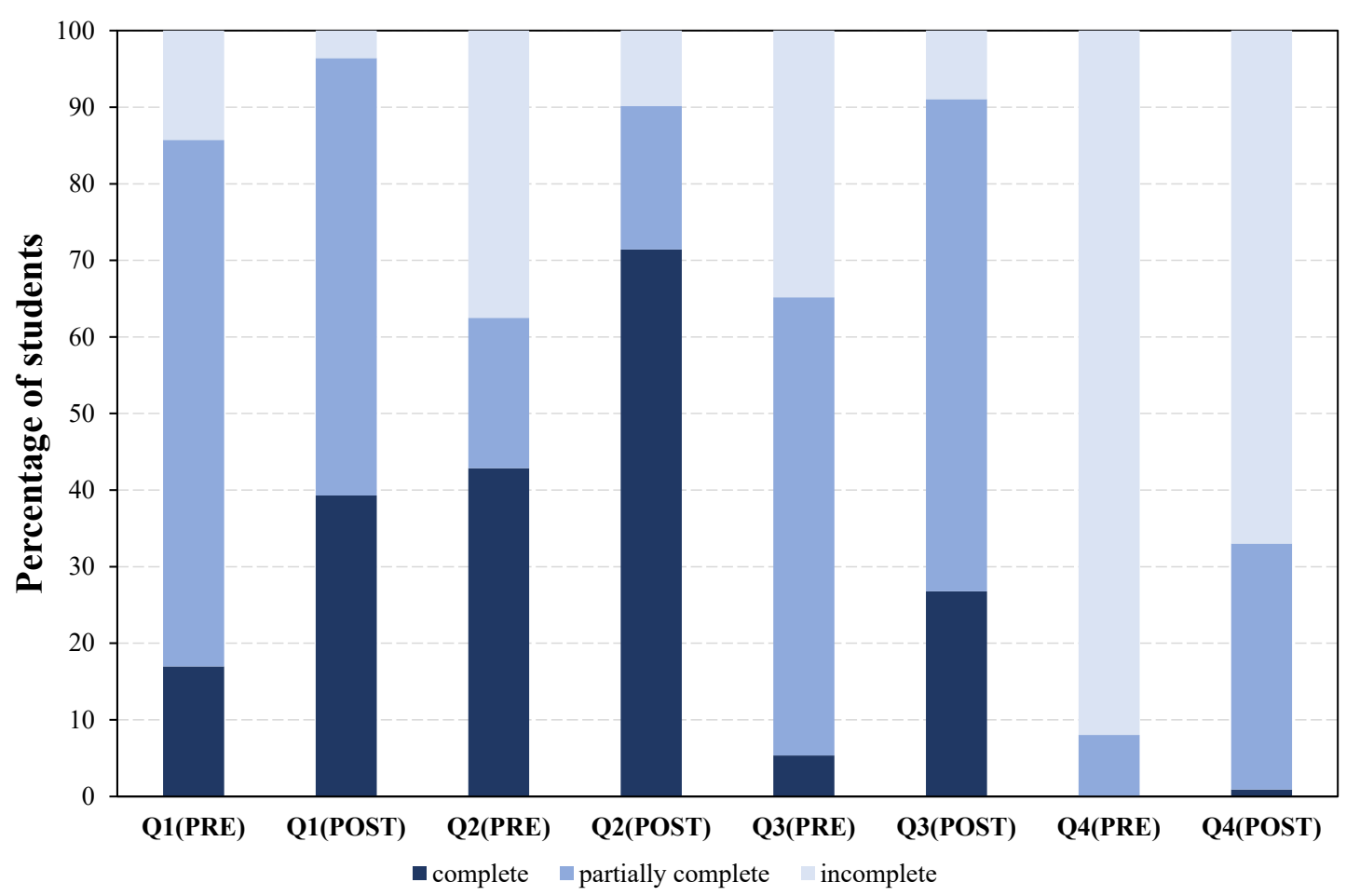

Figure 2. Percentage of answers given by the students in the pre-post questionnaire

Table 4. Statistical significance and size effect for each question of the pre-post questionnaire

\begin{tabular}{lcccc}
\hline Question & $p$ & Statistically significant difference $(\mathrm{p}<0.05)$ & \multicolumn{2}{c}{ Size effect } \\
\hline Q1 & 0.00011 & yes & 0.29 & medium \\
Q2 & $1.64 \mathrm{e}-08$ & yes & 0.39 & medium \\
Q3 & $9.89 \mathrm{e}-08$ & yes & 0.37 & medium \\
Q4 & $3.19 \mathrm{e}-05$ & yes & 0.30 & medium \\
\hline
\end{tabular}

a logarithmic scale") increases very slightly (from 0 to $0.9 \%)$.

In order to check whether the differences observed between the pre-test and the post-test for each question of the questionnaire are statistically significant, the nonparametric Stuart-Maxwell test was used. Table 4 shows the statistical significance and size effect for each question of the pre-post questionnaire. There are statistically significant differences for each question with values of the $\mathrm{r}$ size effect between 0.3 and 0.4 , which indicate a moderate effect (Rosenthal, 1994). Therefore, there has been a significant improvement when carrying out the practical experience on noise pollution based on the use of smartphones for every aspect considered in the questionnaire, especially those related with the sources and effects of noise pollution.

\section{Evaluation of the Practical Experience by Students of the Master in Secondary Education Teaching}

The questionnaire to evaluate the practical experience on noise pollution based on the use of smartphones by the students of the "physics and chemistry" specialisation of the master in secondary education teaching has three scale questions (from 0, strongly disagree, to 10 , strongly agree) and three freeresponse questions.

Regarding the methodological aspects of the proposed practical experience (P1), in general, the master's students strongly agree with each of the six items of the question, obtaining mean scores higher than 7.5 in every one of them (Table 5). The best valued aspects, with mean scores of 9.3 and 9.2 respectively, are that the proposal encourages the students' participation (item 5) and that it shows STS relationships (item 4). Other methodological aspects of the practical experience that have been highly valued are that it helps to acquire scientific knowledge (item 2) and that it helps students to become familiar with scientific methodology (item 3 ), with a mean score of 8.5 , and that it increases the students' interest in science (item 6), with a mean score of 8.2. The worst valued methodological aspect is that which refers to the detection and correction of errors, with a mean score of 7.8.

The master's students strongly agree too with the five items of the question about the contents of the proposal (P2), obtaining mean scores higher than 7 in every one of them (Table 5). The best valued aspect, with a mean score of 9.0, is that the proposal links theory and practice (item $3)$. Other aspects about the contents of the practical 
Table 5. Mean score (and standard deviation) for each item of questions P1 (methodological aspects), P2 (contents) and P6 (overall proposal) of the questionnaire for the students of the "physics and chemistry" specialisation of the master in secondary education teaching

\begin{tabular}{|c|c|c|c|c|c|}
\hline P1 item & mean (SD) & P2 item & mean (SD) & P6 item & mean (SD) \\
\hline 1 & $7.8(1.2)$ & 1 & $7.3(1.6)$ & 1 & $1.6(1.6)$ \\
\hline 2 & $8.5(0.9)$ & 2 & $8.8(1.1)$ & 2 & $2.9(1.9)$ \\
\hline 3 & $8.5(1.0)$ & 3 & $9.0(1.1)$ & 3 & $1.2(1.4)$ \\
\hline 4 & $9.2(1.1)$ & 4 & $8.7(1.2)$ & 4 & $2.6(2.0)$ \\
\hline 5 & $9.3(1.2)$ & 5 & $8.4(1.3)$ & & \\
\hline 6 & $8.2(1.2)$ & & & & \\
\hline
\end{tabular}

experience that have been highly valued are that it adequately presents the concept of noise pollution (item 2 ), with a mean score of 8.8 , that it adequately explains the relationship between sound intensity levels measured at different distances (item 4), with a mean score of 8.7 , and that it adequately presents the concept of decibel (item 5), with a mean score of 8.4. The worst valued aspect about the proposal's contents is the one referring to whether it takes into account the students' previous ideas about waves and sound (item 1), with a mean score of 7.3.

When asked about positive or negative aspects of the proposal on noise pollution with smartphones (P3), the master's students highlighted as positive aspects that "it helps to understand the concepts in a more dynamic and enjoyable way" (15 responses), "it motivates students with the use of smartphones" (14 responses), "it shows STS relationships" (11 responses), "it uses simple materials" (8 responses) and "it is a practical experience" (5 answers). As negative aspects, they mainly highlighted that the proposed practical experience "is difficult to carry out with large groups of students" (10 responses), "the use of smartphones in high schools can be an issue" (9 responses), "more theoretical content is needed about waves and sound in the proposal" (7 answers) and "more time is needed" (5 answers).

When asked about which activities the master's students would eliminate or change from the proposal (P4) and what kind of activities would they use to replace them (P5), there was less consensus. Some of the proposed changes included "making fewer repetitions of the measurements" (4 answers), "eliminating the creation of the sound map of the school" (4 answers), "including a motivating introduction at the beginning of the practical experience" (3 answers) or "making measurements in more locations" (2 answers). The different activities proposed by the master's students included "making measurements outside the school" (20 responses), "making measurements of daily activities (listening to music, watching television, etc.)" (5 answers) or "proposing an activity to check the students' previous ideas on the subject" (2 answers).

Finally, the master's students strongly disagree with each of the four items of the question about general aspects of the proposal (P6), obtaining mean scores lower than 3 in every one of them (Table 5). The items valued with a lower score, 1.2 and 1.6 respectively, are those that state that the topic of the practical experience is not related with the students' reality (item 3) and that it can be eliminated from the secondary education curriculum (item 1). The items valued with a higher score, 2.6 and 2.9 respectively, which still express a strong disagreement, are those that state that the topic of the practical experience is not linked with the other Physics topics studied in the Secondary Education (item 4) and that the theory of this topic is very complicated (item 2).

\section{CONCLUSIONS}

In order to improve the students' motivation and interest towards Physics, practical experiences based on the use of the ubiquitous smartphones as measurement tools have been lately proposed. Thus, a practical experience on noise pollution based on the use of smartphones was designed to help students to better understand the concepts related to this phenomenon. The proposed activities allow students to calibrate the smartphone's sound meter, check the sound attenuation with the square of the distance, use their smartphones to measure sound intensity levels in different places to create a sound map of their school and surrounding area, and draw conclusions though a group discussion about their results and the issue of noise pollution.

First, a diagnostic of the students' knowledge about sound was carried out with a questionnaire which was filled in by 112 students of the $1^{\text {st }}$ year of noncompulsory high school. Results show important shortcomings in the students' knowledge about this phenomenon: they associate sound with a wave, although without specifying which type; they know that sound needs a medium to travel through; they know that ultrasound cannot be detected by humans, although they cannot relate it to the range of frequencies that can be heard by the human ear. Besides, students hardly know any technological applications of sound and they have trouble relating sound qualities with real sounds such as those from musical instruments. These results show the effects that the elimination of this topic from the current Spanish secondary education compulsory curriculum has on the students' knowledge about sound since they will not study this phenomenon until they are in the $2^{\text {nd }}$ year of non-compulsory high school, and only those that choose physics as an optional subject. 
An evaluation of the proposed practical experience was also carried out with a pre-post questionnaire, which was filled in by the same 112 students of $1^{\text {st }}$ year of non-compulsory high school, to check whether this proposal helps students to better understand the concepts of sound and noise pollution. Results show a statistically significant improvement between the global means of the pre-post questionnaire with a large size effect, which indicates that the proposed practical experience on noise pollution with smartphones helps to improve the students' learning about this phenomenon. Besides, statistically significant differences are also observed for each of the questions in the pre-post questionnaire, with a moderate size effect. Therefore, results have confirmed that the proposed practical experience can help students to improve their knowledge about what noise pollution is, its sources and effects, as well as the decibel unit of measurement. Nevertheless, there is still plenty of work to be done since, although in the literature published in recent years there are numerous proposals of practical experiences based on the use of smartphones and/or tablets, many of them have not been developed as specific Physics teaching proposals nor has its effectiveness in the students' learning been evaluated.

Finally, 38 students enrolled in the "physics and chemistry" specialisation of the master in secondary education teaching evaluated the proposed practical experience on noise pollution with smartphones. The proposal was highly valued, with the best considered aspects being that it encourages the students' participation, it links theory and practice, and it shows STS relationships. Thus, the proposed practical experience not only improves the high school students' knowledge about noise pollution, but also allows them to think about the effects that science and technology have on society, a necessary aspect in the education of citizens who are expected to be responsible and respectful with the environment.

Author contributions: All authors have sufficiently contributed to the study, and agreed with the results and conclusions.

Funding: This work was funded jointly by the Spanish Ministry of Science, Innovation and Universities and the European Regional Development Fund through the project PID2019-105320RB-I00.

Acknowledgements: The authors would like to thank the collaboration of the students and teachers of the high schools and the "Physics and Chemistry" specialisation of the Master in Secondary Education Teaching.

Declaration of interest: No conflict of interest is declared by authors.

\section{REFERENCES}

Andrade, M., Richter, J.-L., \& Gutschank, J. (2014). Smartphones in science teaching. Science on Stage Europe. https://www.science-onstage.eu/sites/default/files/material/smartphon es_in_science_teaching.pdf
Buongiorno, D., Longo, A., Michelini, M., Pagotto, S., Ricci, D., \& Santi, L. (2019). APPs in sound measurements to gain a school-work experience. Journal of Physics: Conference Series, 1223, 012002. https:// doi.org/10.1088/17426596/1223/1/012002

Esteve, A. R., Benavent, A., \& Solbes, J. (2019). Smartphones y caída libre: Diseño y evaluación de una experiencia práctica [Smartphones and free fall: Design and evaluation of a practical experience]. Didáctica de las ciencias experimentales y sociales, 37, 165-178. https://doi.org/10.7203/ dces.37.15441

George, R. (2006). A cross domain analysis of change in students' attitudes toward science and attitudes about the utility of science. International Journal of Science Education, 28(6), 571-589. https:/ / doi.org/10.1080/09500690500338755

González, M. A., González, M. A., Martín, M. E., Llamas, C., Martínez, O., Vegas, J., Herguedas, M., \& Hernández, C. (2015). Teaching and learning physics with smartphones. Journal of Cases on Information Technology, 17(1), 31-50. https:// doi.org/10.4018/JCIT.2015010103

González, M.A., \& González, M.A. (2016). Smartphones as experimental tools to measure acoustical and mechanical properties of vibrating rods. European Journal of Physics, 37, 045701. https:/ / doi.org/10.1088/0143-0807/37/4/045701

Hasni, A., \& Potvin, P. (2015). Student's interest in science and technology and its relationships with teaching methods, family context and self-efficacy. International Journal of Environmental and Science Education, 10(3), 337-366. https:/ / doi.org/10.12973 /ijese.2015.249a

Hazelkorn, E., Ryan, C., Beernaert, Y., Constantinou, C. P., Deca, L., Grangeat, M., Karikorpi, M., Lazoudis, A., Pintó Casulleras, R., \& Welzel-Breuer, M. (2015). Science education for responsible citizenship. Report to the European Commission of the expert group on science education. European Union. https:/ / op.europa.eu/s/sIch

Hochberg, K., Gröber, S., Kuhn, J., \& Müller, A. (2014). The spinning disc: Studying radial acceleration and its damping process with smartphone acceleration sensors. Physics Education, 49(2), 137-140. https:// doi.org/10.1088/0031-9120/49/2/137

Kuhn, J., \& Müller, A. (2015). iMobilePhysics: possibilities and limits of using smartphone and tablet-PC as experimental tools. MPTL 2015, Invited Symposium iMP, contribution A41.

Kuhn, J., \& Vogt, P. (2013a). Smartphones as experimental tools: Different methods to determine the gravitational acceleration in classroom physics by using everyday devices. European Journal of Physics Education, 4(1), 16-27. 
Kuhn, J., \& Vogt, P. (2013b). Analyzing acoustic phenomena with a smartphone microphone. The Physics Teacher, 51, 118-119. https://doi.org/10.1119/1.4775539

Kuhn, J., Vogt, P., \& Hirth, M. (2014). Analyzing the acoustic beat with mobile devices. The Physics Teacher, 52 ,

248-249. https://doi.org/10.1119/1.4868948

Linder, C. J. (1992). Understanding sound: So what is the problem? Physics Education, 27, 258-264. https: / / doi.org/10.1088/0031-9120/27/5/004

Merino, J. M. (1998). Some difficulties in teaching the properties of sounds. Physics Education, 33(2), 101104. https:/ / doi.org/10.1088/0031-9120/33/2/014

Monteiro, M., Stari, C., Cabeza, C., \& Martí, A. C. (2017). The polarization of light and Malus' law using smartphones. The Physics Teacher, 55, 264-266. https://doi.org/10.1119/1.4981030

Murphy, C., \& Beggs, J. (2006). Children perceptions of school science. School Science Review, 84(308), 109116.

Organisation for Economic Co-operation and Development (2006). Evolution of student interest in science and technology studies policy report. https://www.oecd.org/science/scitech/36645825.pdf

Osborne, J., \& Dillon, J. (2008). Science education in Europe: critical reflections (vol. 13). The Nuffield Foundation. http:/ / efepereth.wdfiles.com/local--files/scienceeducation/Sci_Ed_in_Europe_Report_Final.pdf

Osborne, J., Driver, R., \& Simon, S. (1998). Attitudes to science: Issues and concerns. School Science Review, $79,27-33$.

Parkinson, J., Hendley, D., Tanner, H., \& Stables, A. (1998). Pupils' attitudes to science in key stage 3 of the national curriculum: A study of pupils in South Wales. Research in Science E Technological Education, 16(2), 165-176.

https: / / doi.org/10.1080/0263514980160206

Parolin, S. O., \& Pezzi, G. (2013). Smartphone-aided measurements of the speed of sound in different gaseous mixtures. The Physics Teacher, 51(8), 508509. https://doi.org/10.1119/1.4824957

Parolin, S. O., \& Pezzi, G. (2015). Kundt's tube experiment using smartphones. Physics Education, 50(4), 508-509. https://doi.org/10.1088/00319120/50/4/443

Perales Palacios, F. J. (1997). Escuchando el sonido: concepciones sobre acústica en alumnos de distintos niveles educativos [Listening to sound: conceptions about acoustics in students of different educational levels]. Enseñanza de las Ciencias, 15(2),
233-247.

https://doi.org/10.5565/rev/ensciencias.4179

Potvin, P., \& Hasni, A. (2014). Analysis of the decline in interest towards school science and technology from Grades 5 through 11. Journal of Science Education and Technology, 23, 784-802. https:/ / doi.org/10.1007/s10956-014-9512-x

Ramsden, J. M. (1998). Mission impossible?: Can anything be done about attitudes to science? International Journal of Science Education, 20(2), 125137. https:/ / doi.org/10.1080/0950069980200201

Rocard, M., Csermely, P., Jorde, D., Lenzen, D., WalbergHenriksson, H., \& Hemmo, V. (2007). Science education NOW: A renewed pedagogy for the future of Europe. Report to the European Commission of the expert group on science education. European Union. https:/ / op.europa.eu/s/sIc1

Rosenthal, R. (1994). Parametric measures of effect size. In H. Cooper, \& L. V. Hedges (Eds.), The handbook of research synthesis (pp. 231-244). Russell Sage Foundation.

Sans, J. A., Gea-Pinal, J., Giménez, M. H., Esteve, A. R., Solbes, J., \& Monsoriu, J. A. (2017). Determining the efficiency of optical sources using a smartphone's ambient light sensor. European Journal of Physics, 38(2), 025301. https:// doi.org/10.1088/13616404/aa51a9

Simpson, R. D., \& Oliver, J. E. (1990). A summary of major influences on attitude toward and achievement in science among adolescent students. Science Education, 74(1), 1-18. https://doi.org/10.1002/sce.3730740102

Sitar, D. (2012). Imaging emission spectra with handheld and cellphone cameras. The Physics Teacher, 50(9), 524-525. https:/ / doi.org/10.1119/1.4767480

Vieyra, R. E., \& Vieyra, C. (2014). Analyzing forces on amusement park rides with mobile devices. The Physics Teacher, 52, 149-151. https: / / doi.org/10.1119/1.4865516

Vogt, P., \& Kuhn, J. (2012a). Analyzing free fall with a smartphone acceleration sensor. The Physics Teacher, 50, 182-183. https:/ / doi.org/10.1119/1.3685123

Vogt, P., \& Kuhn, J. (2012b). Analyzing simple pendulum phenomena with a smartphone acceleration sensor. The Physics Teacher, 50, 439-440. https:/ / doi.org/10.1119/1.4752056

Vogt, P., \& Kuhn, J. (2014). Analyzing collision processes with the smartphone acceleration sensor. The Physics Teacher, 52, 118-119. https: / / doi.org/10.1119/1.4862122

\section{http://www.ejmste.com}

